\title{
Impact of Interhospital Transfer on Outcomes in Non-emergency Colorectal Surgery
}

\author{
Stephen P. Sharp ${ }^{1} \cdot$ Daniel J. Schuster $^{1} \cdot$ Ashar Ata $^{1} \cdot$ Brian T. Valerian $^{1}$ \\ Jonathan J. Canete ${ }^{1} \cdot$ A. David Chismark ${ }^{1} \cdot$ Edward C. Lee $^{1,2}$
}

Published online: 27 October 2017

(C) Société Internationale de Chirurgie 2017

\begin{abstract}
Background A paucity of data exists on the impact of transfer status on outcomes for patients undergoing nonemergency (urgent) colorectal surgery. This study characterized transferred patients undergoing urgent colorectal surgery and determined which patient comorbidities significantly contributed to poor outcomes.

Methods The American College of Surgeons National Surgical Quality Improvement Program database from 2012 to 2013 was used. Urgent direct admissions undergoing colon, rectum, or small bowel operations were compared to urgent transfers using bivariate and multivariable analysis models. Primary outcomes were overall complications, hospital length of stay, and mortality.

Results A total of 82,151 admissions were analyzed. After multivariable analysis, direct admission patients had nearly similar risk of complications ( $\mathrm{RR}=0.95 ; 95 \% \mathrm{CI} 0.91-0.99)$ and length of hospital stay (7\% shorter; $95 \% \mathrm{CI}$ 4-9\%), as well as no difference in mortality ( $\mathrm{RR}=0.94 ; 95 \%$ CI $0.80-1.11)$.

Conclusions Transfer status alone confers minimal risk toward higher complication rates and longer hospital length of stay in patients undergoing urgent colorectal surgery, and the poor outcomes observed in this cohort are largely due to patient comorbidities and disease severity. Our results suggest that outcomes in transferred colorectal surgery patients undergoing urgent operations depend mainly on operative acuity and clinical factors, and to a lesser degree transfer status.
\end{abstract}

\section{Introduction}

Interhospital transfer has become increasingly common over the previous decade. The typical transferred patient has more complex medical and surgical pathology, and

Presented as e-Poster of Distinction at The American Society of Colon and Rectal Surgeons Annual Scientific Meeting Los Angeles, CA, May 2nd 2016.

Edward C. Lee

LeeE@mail.amc.edu

1 Division of Colon and Rectal Surgery, Albany Medical Center, 43 New Scotland Avenue, Albany, NY 12208, USA

250 New Scotland Avenue, MC-193, 6th Floor, Albany, NY 12208, USA frequently these patients have higher complication rates and worse outcomes compared to patients admitted directly from home or the emergency department of the accepting institution. Furthermore, there has been an increased emphasis on pay-for-performance status and tiered reimbursement schemes which do not typically account for hospital transfer status [1, 2]. Recently, there have been multiple studies with conflicting results evaluating the impact of interhospital transfer in surgical outcomes [3, 4].

For emergency operations, Lucas et al. demonstrated similar outcomes between transferred and directly admitted patients after adjusting for patient comorbidities [3], which were consistent with our previous study [5]. However, our results indicated that for non-emergency (urgent) operations, transferred patients have worse outcomes compared to direct admissions even after propensity score matching 
[5]. Importantly, the urgent transfer group included a heterogeneous mix of elective and urgent cases. Recently, the American College of Surgeons (ACS) National Surgical Quality Improvement Program (NSQIP) has limited the coding of transferred cases to either emergency or nonemergency (urgent) to more clearly define these subgroups, while direct admissions could be coded as emergency, nonemergency, or elective [6].

Given the coding problems involving the urgent transfer cohort from previous studies, the objectives of this study were (1) to better characterize the urgent transfer group in the recently updated NSQIP cohort (2012-2013); (2) to compare complication rates, mortality, and hospital length of stay between urgent transfers and urgent direct admissions. Furthermore, as elective cases are generally used to benchmark patient outcomes, we sought (3) to determine the absolute differences in complication rates, mortality, and hospital length of stay between urgent transfers and elective direct admissions; and finally (4) to identify the patient factors and comorbidities that contribute significantly to higher complication rates, increased mortality, and longer hospital stay in patients undergoing colorectal surgery.

\section{Materials and methods}

This was an institutional review board-approved, retrospective cohort study using the ACS-NSQIP database. NSQIP is a nationally validated, risk-adjusted, outcomesbased program to measure and improve the quality of surgical care. The NSQIP database is comprised of over 150 variables, including patient demographics, preoperative risk factors, intra-operative variables, and 30-day postoperative morbidity and mortality outcomes for a sample of patients undergoing major surgical procedures at participating hospitals [6]. A surgical clinical reviewer records de-identified patient demographic information, comorbidities, laboratory results, intra-operative details, postoperative occurrences, and 30-day mortality and hospital readmission [7].

The study population was limited to colon, rectum, and small bowel operations from 2012 to 2013. While small bowel procedures would seem to fall outside the realm of colorectal surgery, these procedures were included due to the frequent involvement of the small bowel with colorectal pathology, i.e., Crohn's disease, mesenteric ischemia, and other complex abdominal pathology. Current procedural terminology (CPT) codes and procedures are listed in Table 1. For the transfer cohort, the "urgent" category was defined as being non-emergency cases; and for the direct admission cohort, "urgent" was defined as cases that were both non-elective as well as non-
Table 1 Intestinal surgery current procedural terminology codes

\begin{tabular}{|c|c|}
\hline \multirow{5}{*}{$\begin{array}{l}\text { Small } \\
\text { bowel }\end{array}$} & 44005-enterolysis \\
\hline & $\begin{array}{l}44120,44121,44125,44202,44203 \text {-small bowel } \\
\text { resection }\end{array}$ \\
\hline & 44615-strictureplasty \\
\hline & 44620 -ileostomy reversal \\
\hline & $44340,44310,44312,44316$-ileostomy \\
\hline \multirow[t]{17}{*}{$\begin{array}{l}\text { Colon/ } \\
\text { rectum }\end{array}$} & $\begin{array}{l}\text { 44139, 44140, 44141, 44143, 44144, 44204, 44206, } \\
\text { 44625-segmental colectomy }\end{array}$ \\
\hline & $\begin{array}{l}44145,44146,44207,44208,44147,45119,45120- \\
\text { low anterior resection }\end{array}$ \\
\hline & 44210,44150 - total abdominal colectomy \\
\hline & $\begin{array}{l}44212,44151,44156,45121,45123,44155 \text {-total } \\
\text { proctocolectomy }\end{array}$ \\
\hline & 45113-proctectomy with ileal pouch-anal anastomosis \\
\hline & 45110 -abdominal perineal resection \\
\hline & 44205,44160 -ileocolic resection \\
\hline & 44604, 44605-colorrhaphy \\
\hline & 44626 - colostomy reversal \\
\hline & 45126 - pelvic exenteration \\
\hline & $44346,44345,44314,44322,44320$ —colostomy \\
\hline & 45160,45170 - transanal excision of rectal lesion \\
\hline & $45550,45541,45540$-rectopexy \\
\hline & $\begin{array}{l}46750,46753,46760,46761,46762,46924,46940, \\
\quad 46942,46715,46705,46700,45999,45910,45905, \\
\text { 45825, 45820, 45560, 45108, 45130, 45190- } \\
\text { anorectal procedure, other }\end{array}$ \\
\hline & $\begin{array}{l}\text { 46706, 46060, 46030, 46020, 57308-treatment of anal } \\
\text { fistula }\end{array}$ \\
\hline & $\begin{array}{l}46045,46040,45000,45005,45020 \text {-incision and } \\
\text { drainage of anorectal abscess }\end{array}$ \\
\hline & 46947, 45505-excisional hemorrhoidectomy \\
\hline
\end{tabular}

emergency. Both laparoscopic and open cases were included. Transferred patients were defined as coming from an inpatient acute care hospital or an outside emergency department. Patients coming from skilled nursing facilities, chronic care facilities, and unidentified facilities were excluded. The direct admission cohort was admitted from home or directly from the emergency department. All transferred patients received an operation at the receiving facility, and NSQIP does not differentiate which patients did and did not receive an operation at the transferring facility.

Preoperative patient demographics considered as potential confounders included age, sex, body mass index (BMI), American Society of Anesthesiologists (ASA) risk class, transfer status, smoking status, functional status, presence of sepsis, $>10 \%$ loss of body weight over previous 6 months, and preoperative blood transfusion $>1$ unit. Comorbidities of interest included chronic obstructive pulmonary disease (COPD), dyspnea, hypertension, 
congestive heart failure (CHF), insulin-dependent diabetes, end-stage renal disease (ESRD) requiring dialysis, steroid dependence, and bleeding disorder. Laboratory factors of interest included white blood cell (WBC) count, hematocrit (HCT), creatinine $(\mathrm{Cr})$, and albumin levels. Operative factors included type of procedure and operative time.

Outcomes of interest included major postoperative complications, mortality, and total hospital length of stay (HLOS). Major postoperative complications included surgical site infection, wound dehiscence, pneumonia, unplanned intubation, prolonged ventilator requirement $>48 \mathrm{~h}$, elevated transfusion requirement, pulmonary embolism, deep vein thrombosis, urinary tract infection, pneumonia, CHF, myocardial infarction, acute kidney injury, stroke, sepsis, and septic shock.

\section{Statistical analysis}

Patient demographics, comorbidities, surgery type, and major postoperative complications were compared by transfer status using Chi-square or $t$ tests, as appropriate. Bivariate and multivariable analyses were performed in order to control for patient factors and comorbidities. Analysis was performed on patients who had complete data for all variables of interest. Missing data comprised less than $5 \%$ of the original cohort and was imputed. Modified Poisson regression and robust standard errors were used to estimate the risk ratios associated with various risk factors for mortality and morbidity [8]. Hospital length of stay was log transformed and calculated as the ratio of mean difference, and the effect of various risk factors was assessed using linear regression [9]. Variables significant at 0.10 level of significance were considered for adjustment in the multivariable analysis. All statistical analyses were performed using Stata software, version 14.0 (Stata Corp), using a $p$ value of 0.05 for two-tailed tests as a cutoff for statistical significance.

\section{Results}

\section{Demographics}

The demographic data for the two groups are presented in Table 2. A total of 82,151 colorectal surgery patients were queried from the 2012-2013 ACS-NSQIP database. Totally, 79,835 (97\%) were directly admitted and 2316 (3\%) cases were transferred. Urgent transfers and urgent direct admissions had similar age classification and BMI. Urgent transfers had slightly higher rates of impaired functional status (10.8 vs. $8.2 \%$ ) and higher ASA classification (76.5 vs. $69.9 \%)$. Urgent transfer patients also tended to have slightly higher rates of COPD, CHF, chronic steroid use, bleeding disorders, anemia, and insulin-dependent diabetes.

\section{Complications}

Urgent transfers had higher complication rates than urgent direct admissions as well as elective direct admissions (Table 3). Common complications included hematologic derangements: need for transfusion, deep vein thrombosis, and pulmonary embolism; surgical site infections (SSI): superficial SSI, organ space SSI, deep SSI, and wound dehiscence; infectious complications: sepsis, urinary tract infection, and septic shock; respiratory complications: pneumonia, re-intubation, and prolonged ventilator requirements. Other complications that were slightly higher in the urgent transfer cohort included cardiac arrest and acute renal failure.

\section{Surgical outcomes}

Risk ratios for any complication, mortality, and HLOS were calculated using univariate analysis using Stata 14.0 (Fig. 1). On bivariate analysis, urgent direct admissions had a lower risk of complications compared to urgent transfers $(\mathrm{RR}=0.79 ; p$ value $<0.001)$, lower mortality $(\mathrm{RR}=0.71 ; p$ value $<0.001)$, as well as shorter HLOS (17\% shorter; $p$ value $<0.001)$.

However, after multivariable analysis, transfer status had a small impact on complication rates in the urgent cohort $(\mathrm{RR}=0.95 ; p$ value 0.03$)$ and no significant impact on mortality $(\mathrm{RR}=0.94 ; p$ value 0.49$)$ or $\operatorname{HLOS}(7 \%$ shorter; $p$ value 0.47 ). Furthermore, elective direct admissions had significantly lower rates of poor outcomes compared to urgent transfers even after multivariable analysis (complications: $\mathrm{RR}=0.77 ; p$ value $<0.001$; mortality: $\mathrm{RR}=0.59 ; p$ value $<0.001$; length of stay: $60 \%$ shorter; $p$ value $<0.001$ ).

\section{Clinical predictors of increased complication rates}

The main clinical risk factors associated with increased complication rates after multivariable analysis are presented in Fig. 2a (GraphPad Prism 7). The most significant risk factors were Hct $<30$ and sepsis. Intermediate risk factors were higher ASA classification, totally dependent functional status, ascites, and presence of metastatic cancer.

\section{Clinical predictors of increased mortality rates}

Significant risk factors associated with higher mortality rates after multivariable analysis are presented in Fig. $2 b$ (GraphPad Prism 7). The most significant risk factors were 
Table 2 Baseline cohort demographics and characteristics

\begin{tabular}{|c|c|c|c|c|}
\hline & $\begin{array}{l}\text { Urgent transfer } \\
(N=2316) \\
\%\end{array}$ & $\begin{array}{l}\text { Urgent direct admission } \\
(N=14,337) \\
\%\end{array}$ & $\begin{array}{l}\text { Elective direct admission } \\
(N=65,498) \\
\%\end{array}$ & $p$ value \\
\hline Female & 53.9 & 53.6 & 51.4 & $*$ \\
\hline \multicolumn{5}{|l|}{ Age } \\
\hline $18-30$ years & 6.6 & 4.8 & 3.9 & $*$ \\
\hline $31-40$ years & 6.7 & 6.1 & 6.4 & $*$ \\
\hline $41-50$ years & 10.7 & 11.4 & 13.2 & $*$ \\
\hline $51-60$ years & 20.4 & 19.3 & 24.7 & $*$ \\
\hline $61-70$ years & 22.1 & 22.9 & 26.1 & $*$ \\
\hline 71-90 years & 33.5 & 35.6 & 25.7 & $*$ \\
\hline \multicolumn{5}{|l|}{ ASA class } \\
\hline $1-2$ & 23.2 & 29.9 & 51.9 & $*$ \\
\hline $3-5$ & 76.5 & 69.9 & 48.0 & $*$ \\
\hline \multicolumn{5}{|l|}{ BMI } \\
\hline Underweight & 6.7 & 6.1 & 2.8 & $*$ \\
\hline Normal & 35.8 & 35.6 & 30.2 & $*$ \\
\hline Overweight & 25.9 & 29.7 & 33.3 & $*$ \\
\hline Obese & 24.4 & 23.1 & 28.4 & $*$ \\
\hline Morbidly obese & 7.2 & 5.5 & 5.3 & $*$ \\
\hline $\begin{array}{l}\text { Impaired functional } \\
\text { status }\end{array}$ & 10.8 & 8.2 & 1.7 & $*$ \\
\hline Current smoker & 22.4 & 20.0 & 17.6 & $*$ \\
\hline Dialysis & 2.3 & 2.0 & 0.5 & $*$ \\
\hline Wound infection & 9.3 & 5.6 & 2.0 & $*$ \\
\hline Hypertension & 50.1 & 51.5 & 46.6 & $*$ \\
\hline Insulin use & 9.2 & 7.4 & 4.5 & $*$ \\
\hline COPD & 10.2 & 8.0 & 4.5 & $*$ \\
\hline Congestive heart failure & 4.2 & 2.4 & 0.4 & $*$ \\
\hline Chronic steroid use & 14.9 & 10.9 & 7.1 & $*$ \\
\hline Bleeding disorder & 12.1 & 8.9 & 2.6 & $*$ \\
\hline Hct $<31$ & 36.6 & 27.7 & 7.4 & $*$ \\
\hline Hct $31-45$ & 60.5 & 67.7 & 78.2 & $*$ \\
\hline Het $>45$ & 2.4 & 4.0 & 9.1 & $*$ \\
\hline $\mathrm{Cr}<1.19$ & 81.6 & 83.6 & 79.7 & $*$ \\
\hline $\mathrm{Cr}>1.19$ & 17.7 & 15.7 & 12.2 & $*$ \\
\hline $\mathrm{WBC}<4$ & 5.4 & 5.6 & 4.9 & $*$ \\
\hline WBC 4-11 & 67.2 & 72.2 & 81.4 & $*$ \\
\hline $\mathrm{WBC}>11$ & 26.7 & 21.6 & 7.0 & $*$ \\
\hline Albumin $>3.5$ & 21.3 & 39.8 & 52.8 & $*$ \\
\hline Albumin $<3.5$ & 65.5 & 48.1 & 11.1 & $*$ \\
\hline
\end{tabular}

$* p$ value $<0.001$

higher ASA classification and metastatic cancer. Intermediate risk factors were age $>70$, totally dependent functional status, and ascites.

\section{Clinical predictors of extended hospital length of stay}

Clinical predictors associated with extended hospital length of stay (defined as $>9$ days length of stay) after multivariable analysis are presented in Fig. 2c (GraphPad Prism 
Table 3 Outcomes and complication rates by transfer and acuity status

\begin{tabular}{|c|c|c|c|c|c|}
\hline Outcomes & $\begin{array}{l}\text { Urgent transfer } \\
(N=2316)\end{array}$ & & $\begin{array}{l}\text { Urgent direct admission } \\
(N=14,337)\end{array}$ & $\begin{array}{l}\text { Elective direct admission } \\
(N=65,498)\end{array}$ & $p$ value \\
\hline Overall morbidity (\%) & 49.9 & & 39.4 & 22.2 & $*$ \\
\hline Overall mortality (\%) & 6.6 & & 4.7 & 0.9 & $*$ \\
\hline $\begin{array}{l}\text { Hospital length of stay } \\
\text { (days) }\end{array}$ & $16.42( \pm 0.26)$ & & $13.88( \pm 0.10)$ & $6.41( \pm 0.03)$ & $*$ \\
\hline Specific complications & & $\%$ & $\%$ & $\%$ & \\
\hline \multicolumn{6}{|l|}{ Hematologic } \\
\hline Transfusion & & 24.8 & 18.5 & 7.7 & * \\
\hline Deep vein thrombosis & & 3.9 & 2.2 & 0.9 & * \\
\hline Pulmonary embolism & & 1.3 & 1.0 & 0.5 & * \\
\hline \multicolumn{6}{|l|}{ Surgical site } \\
\hline Superficial SSI & & 9.1 & 6.8 & 6.2 & * \\
\hline Organ space SSI & & 7.0 & 5.3 & 3.9 & * \\
\hline Deep SSI & & 3.1 & 2.2 & 1.5 & * \\
\hline Wound dehiscence & & 2.6 & 1.7 & 1.1 & * \\
\hline \multicolumn{6}{|l|}{ Cardiac } \\
\hline Myocardial infarction & & 0.9 & 0.8 & 0.4 & * \\
\hline Cardiac arrest & & 1.3 & 0.9 & 0.3 & * \\
\hline \multicolumn{6}{|l|}{ Respiratory } \\
\hline Pneumonia & & 6.2 & 4.5 & 1.5 & * \\
\hline Re-intubation & & 5.0 & 3.4 & 1.2 & * \\
\hline Prolonged vent $>48 \mathrm{~h}$ & & 8.6 & 4.5 & 1.1 & $*$ \\
\hline \multicolumn{6}{|l|}{ Infectious } \\
\hline Sepsis & & 9.6 & 7.5 & 3.1 & * \\
\hline Urinary tract infection & & 5.3 & 4.0 & 2.5 & $*$ \\
\hline Septic shock & & 6.2 & 3.4 & 1.0 & $*$ \\
\hline \multicolumn{6}{|l|}{ Neurologic } \\
\hline Stroke & & 0.3 & 0.5 & 0.2 & $*$ \\
\hline \multicolumn{6}{|l|}{ Renal } \\
\hline ARF & & 1.2 & 0.9 & 0.4 & * \\
\hline
\end{tabular}

$* p$ value $<0.001$

7). There was no difference between urgent transfers and urgent direct admissions. Elective direct admissions had a significantly less risk of extended HLOS compared to urgent transfers $(\mathrm{RR}=0.60 ; p$ value $<0.001)$. The most significant risk factors for extended HLOS in the entire cohort were higher ASA classification and metastatic cancer. Intermediate risk factors were age $>70$ $(\mathrm{RR}=2.33 ; p$ value $<0.001)$, totally dependent functional status $(\mathrm{RR}=2.21 ; p$ value $<0.001)$, and ascites $(\mathrm{RR}=2.09 ; p$ value $<0.001)$.

\section{Discussion}

Interhospital transfer from smaller community hospitals to larger tertiary care centers typically involves patients who are critically ill, have multiple comorbidities, and require complex surgical care. Historically, a majority of the literature encompassing interhospital transfer has involved medicine patients [10] and has shown improved patient outcomes in specialties such as trauma and cardiology $[11,12]$. For patients in the medical intensive care unit, however, Durairaj et al. concluded that transfer status was an independent risk factor for in-hospital mortality [13]. Among acute care surgery patients, Santry et al. found that transferred patients undergoing an emergency operation 

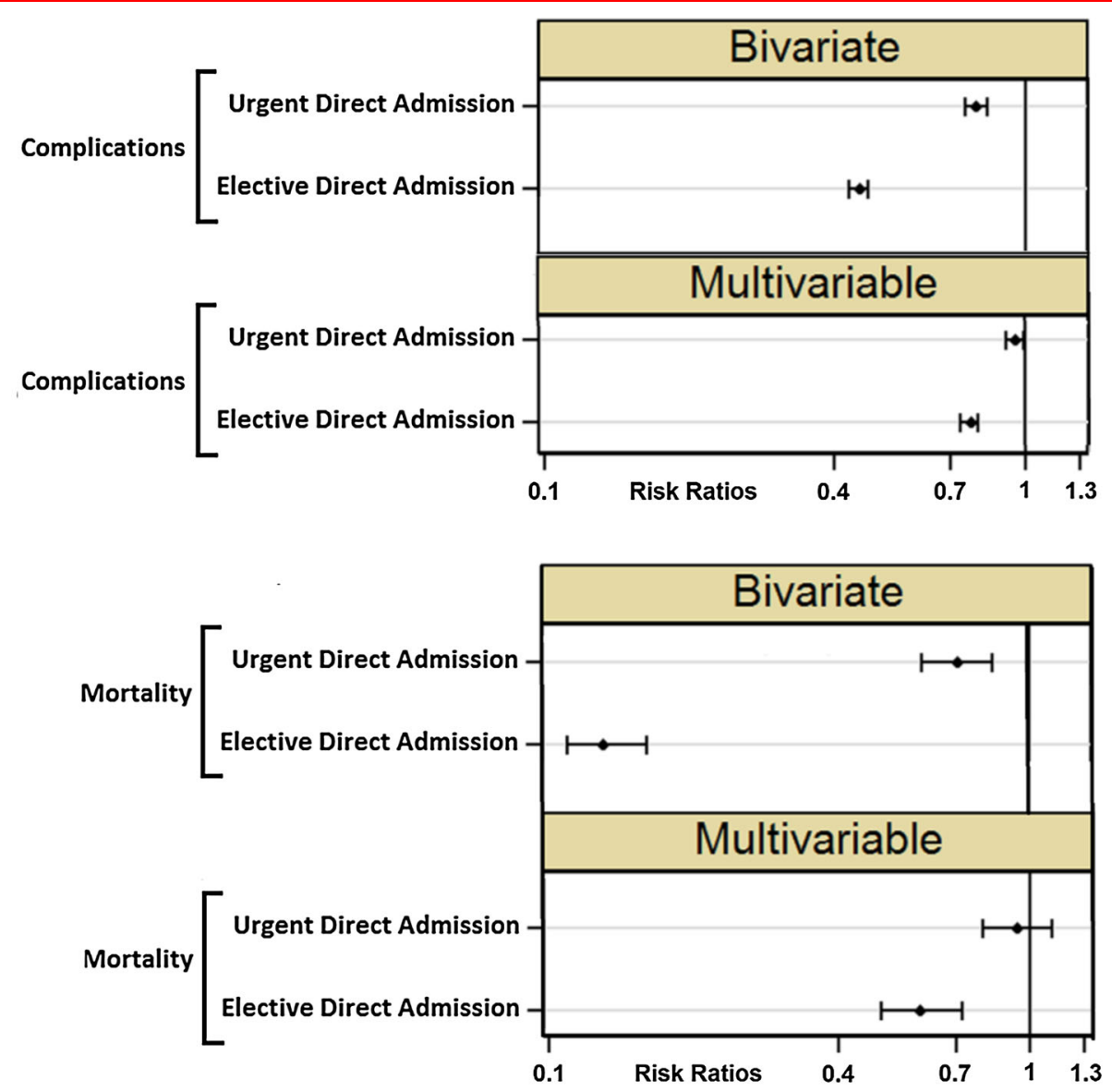

Hospital Length of Stay

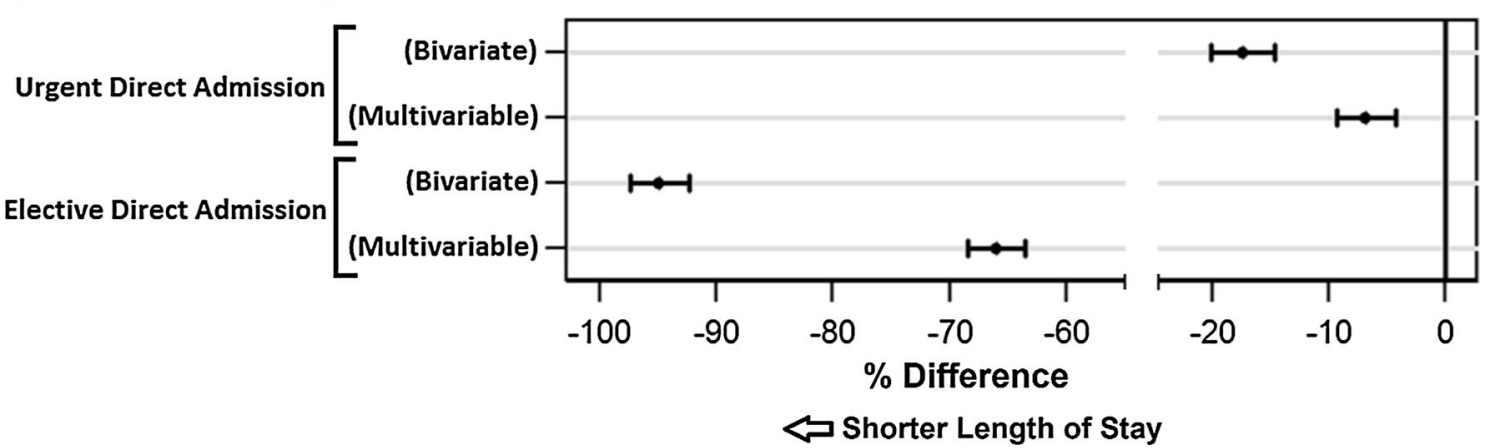

Fig. 1 Surgical outcomes in urgent colorectal surgery transfers versus direct admissions. Bivariate and multivariable surgical outcomes for urgent interhospital transfers versus urgent direct admissions in the overall cohort, and the effect of elective operative status on surgical outcomes

had worse outcomes than directly admitted patients, although this difference was not statistically significant after multivariable analysis [14].

General surgery cases, particularly colorectal surgery cases, comprise a significant percentage of patients involving interhospital transfer. Recently, there have been multiple studies evaluating the impact on surgical outcomes from interhospital transfers, and the results have yielded some conflicting and confusing conclusions. Lucas et al. [3] evaluated a mix of general surgery cases limited to urgent and emergency cases using the ACS-NSQIP database. This study concluded that after multivariable analysis, transfer status alone did not contribute to poor surgical outcomes, which was largely explained by confounding patient factors and comorbidities. Our prior study suggested that among urgent colorectal surgery cases, 

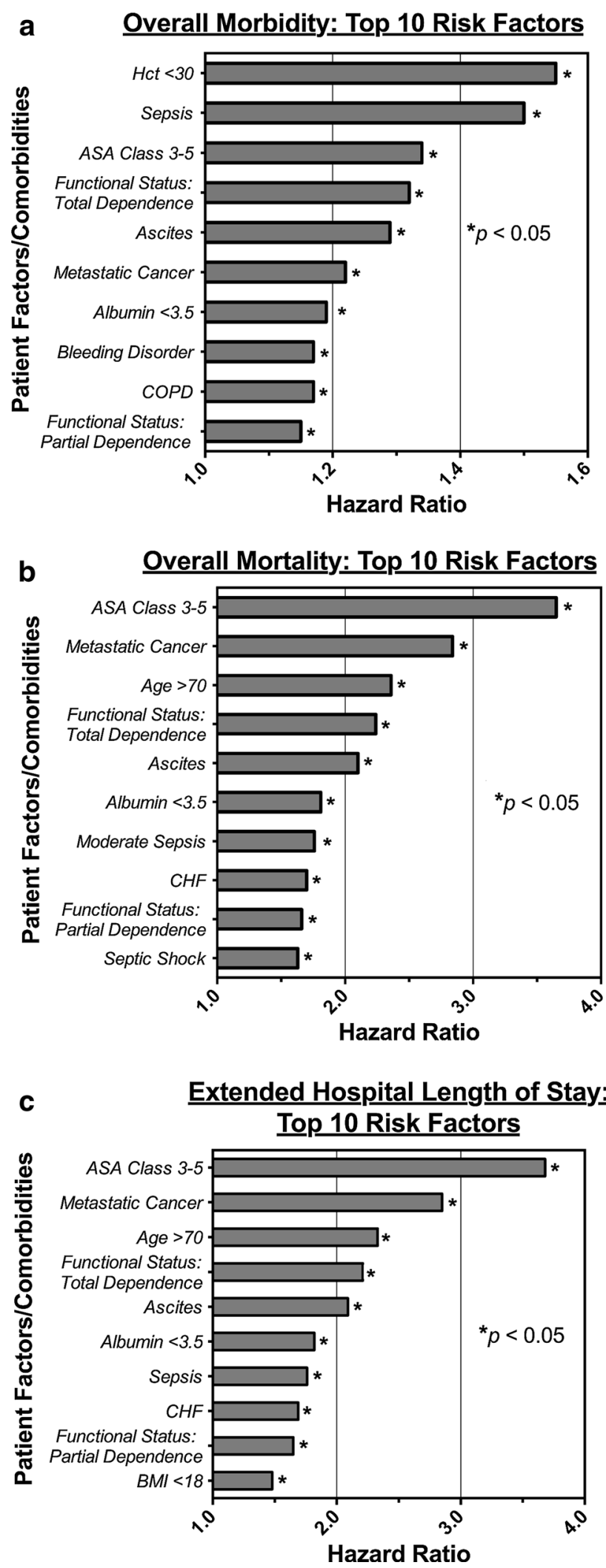

4Fig. 2 a Top 10 risk factors for overall morbidity in urgent colorectal surgery transfers. Hct hematocrit, ASA American Society of Anesthesiologists, $\mathrm{CHF}$ congestive heart failure. b Top 10 risk factors for overall mortality in urgent colorectal surgery transfers. Hct hematocrit, ASA American Society of Anesthesiologists, COPD chronic obstructive pulmonary disease. c Top 10 risk factors for extended hospital length of stay in urgent colorectal surgery transfers. ASA American Society of Anesthesiologists, $C H F$ congestive heart failure, $B M I$ body mass index

transferred patients still experienced higher rates of complications and worse outcomes, even after extensive propensity score matching [5], which was further confirmed by Chow et al. [15]. One of the significant limitations with these studies was that the transfer cohort included a heterogeneous mix of both urgent and elective procedures. Upon further subset analysis, the elective cohort contributed significantly to the higher complication rates, even after multivariable analysis, and this could not be explained. Beginning in 2012, NSQIP limited the coding of transfer patients to either emergency or urgent (nonemergency), which eliminated the elective category. As a result of the updated transfer coding status, we sought to reevaluate and compare the results of the previous study to those under the updated definitions, in order to determine their accuracy and whether or not such a change in coding distinction could ultimately alter the surgical outcomes data. In our prior study, the inclusion of elective cases in both groups likely offsets the poor outcomes in the transfer group. With the new classification system, we feel this provides a more accurate representation of truly urgent cases.

To our knowledge, this is the first ACS-NSQIP study to attempt to elucidate the effect of transfer status in urgent colorectal surgery cases using the updated NSQIP classifications of transferred patients. Our data suggest that transfer status alone confers a small risk toward higher complication rates in patients undergoing urgent colorectal surgery, and the poor outcomes observed in this cohort are largely due to patient comorbidities and disease severity. Our results suggest that outcomes in transferred colorectal surgery patients undergoing urgent operations should be standardized based mainly on operative acuity and clinical factors, and to a lesser degree transfer status. These data highlight the importance of accurate coding of surgical procedures in analyzing surgical outcomes and emphasize the need for more specialty-specific ACS-NSQIP databases. This study highlights the importance of stronger national or international quality improvement databases to track and improve surgical outcomes.

This study highlights some of the inherent limitations with large quality initiatives such as NSQIP, where reliance on administrative data is paramount to track relevant 
outcomes, and how these quality initiatives measure certain postoperative events which may be more relevant in one specialty over another [16, 17]. Furthermore, these results emphasize the importance of specificity and accuracy in coding within NSQIP, and some have suggested that forming specialty-specific NSQIP collaboratives may provide more specific outcome information. Birkmeyer et al. discussed the development of specialty-specific organizations within the ACS-NSQIP constructs to identify patientand operation-specific variables and quality metrics [18]. At our institution, the colorectal surgery department is involved in the IBD-NSQIP collaborative study whereby specific procedure and patient data are collected such as the type of proctocolectomy as well as more specific patient factors such as the type of biologic agent(s) or immunomodulators. Based on the results of our study and how minor classification changes in a large quality initiative database can produce drastically different results and influence practice changes, we propose to establish a similar colorectal surgery group within ACS-NSQIP.

A study of this type has several limitations. First, there is an unavoidable selection bias, and there are multiple reasons to transfer a patient other than for complexity care, such as patient continuity ("the other hospital's patient") or postoperative complications that the transferring facility is unable to treat (such as anastomotic leaks).

\section{Conclusions}

In conclusion, any higher complication rates and longer hospital length of stay in patients undergoing urgent colorectal surgery are predominantly due to patient comorbidities and disease severity and are not significantly altered by transfer status. Our results suggest that outcomes in transferred colorectal surgery patients undergoing urgent operations should be standardized based mainly on operative acuity and clinical factors, and to a lesser degree transfer status. Finally, this study advocates for more specialty- and procedure-specific coding within NSQIP and refined outcome measurements in order to provide more clinically relevant outcomes data and conclusions.

\begin{abstract}
Author's contribution SS, DS and AA contributed to the study design, collected samples, interpreted data, drafted and revised the manuscript. BV, JC, DC and EL contributed to the study design, assisted in drafting and revising the manuscript. All authors read and approved the final manuscript.
\end{abstract}

\section{Compliance with ethical standards}

Conflict of interest The authors declare that they have no conflict of interest.
Ethical approval The authors are in compliance with all applicable ethical standards.

\section{References}

1. Jancin B (2007) Medicare may drop payments for SSI. Gen Surg News 3:1

2. Yi F (2014) The future of quality measurement in the United States. Clin Colon Rectal Surg 27(1):32-38

3. Lucas DJ, Ejaz A, Haut ER, Spolverato G, Haider AH, Pawlik TM (2014) Interhospital transfer and adverse outcomes after general surgery: implications for pay for performance. J Am Coll Surg 218(3):393-400

4. Flabouris A, Hart GK, George C (2008) Outcomes of patients admitted to tertiary intensive care units after interhospital transfer: comparison with patients admitted from emergency departments. Crit Care Resusc 10(2):97-105

5. Sharp SP, Ata A, Valerian BT, Canete JJ, Chismark AD, Lee EC (2016) Complications and surgical outcomes after interhospital transfer vs direct admission in colorectal surgery: a national surgical quality improvement program analysis. Am J Surg 213(6):1031-1037

6. Surgeons ACo (2014) ACS NSQIP. Participant list. http://site. acsnsqip.org/participants/. Access date August 12, 2014

7. Cohen ME, Ko CY, Bilimoria KY et al (2013) Optimizing ACS NSQIP modeling for evaluation of surgical quality and risk: patient risk adjustment, procedure mix adjustment, shrinkage adjustment, and surgical focus. J Am Coll Surg 217(2):336-346

8. Zou G (2004) A modified poisson regression approach to prospective studies with binary data. Am J Epidemiol 159(7):702-706

9. Friedrich JO, Adhikari NK, Beyene J (2008) The ratio of means method as an alternative to mean differences for analyzing continuous outcome variables in meta-analysis: a simulation study. BMC Med Res Methodol 8:32

10. Golestanian E, Scruggs JE, Gangnon RE, Mak RP, Wood KE (2007) Effect of interhospital transfer on resource utilization and outcomes at a tertiary care referral center. Crit Care Med 35(6):1470-1476

11. MacKenzie EJ, Rivara FP, Jurkovich GJ et al (2006) A national evaluation of the effect of trauma-center care on mortality. N Engl J Med 354(4):366-378

12. Cantor WJ, Fitchett D, Borgundvaag B et al (2009) Routine early angioplasty after fibrinolysis for acute myocardial infarction. N Engl J Med 360(26):2705-2718

13. Durairaj L, Will JG, Torner JC, Doebbeling BN (2003) Prognostic factors for mortality following interhospital transfers to the medical intensive care unit of a tertiary referral center. Crit Care Med 31(7):1981-1986

14. Santry HP, Janjua S, Chang Y, Petrovick L, Velmahos GC (2011) Interhospital transfers of acute care surgery patients: Should care for nontraumatic surgical emergencies be regionalized? World J Surg 35(12):2660-2667. doi:10.1007/s00268-011-1292-3

15. Chow CJ, Gaertner WB, Jensen CC, Sklow B, Madoff RD, Kwaan MR (2017) Does hospital transfer impact outcomes after colorectal surgery? Dis Colon Rectum 60(2):194-201

16. Cima RR, Lackore KA, Nehring SA et al (2011) How best to measure surgical quality? Comparison of the Agency for Healthcare Research and Quality Patient Safety Indicators (AHRQ-PSI) and the American College of Surgeons National Surgical Quality Improvement Program (ACS-NSQIP) postoperative adverse events at a single institution. Surgery 150(5):943-949 
17. Lawson EH, Louie R, Zingmond DS et al (2012) A comparison of clinical registry versus administrative claims data for reporting of 30-day surgical complications. Ann Surg 256(6):973-981
18. Birkmeyer JD, Shahian DM, Dimick JB et al (2008) Blueprint for a new American College of Surgeons: national surgical quality improvement program. J Am Coll Surg 207(5):777-782 